OPEN ACCESS

Edited by:

Maurizio Acampa,

Siena University Hospital, Italy

Reviewed by:

Edward Feldmann,

Brown University, United States

Futoshi Mori,

Iwate Medical University, Japan

*Correspondence:

Yongjun Wang

yongjunwang@ncrcnd.org.cn

Specialty section:

This article was submitted to

Stroke,

a section of the journal

Frontiers in Neurology

Received: 18 July 2019

Accepted: 11 December 2019

Published: 17 January 2020

Citation:

Chen Z, Qin H, Liu J, Wu B, Cheng Z, Jiang $Y$, Liu L, Jing L, Leng $X$, Jing J, Wang $Y$ and Wang $Y(2020)$

Characteristics of Wall Shear Stress and Pressure of Intracranial

Atherosclerosis Analyzed by a Computational Fluid Dynamics Model: A Pilot Study. Front. Neurol. 10:1372. doi: 10.3389/fneur.2019.01372

\section{Characteristics of Wall Shear Stress and Pressure of Intracranial Atherosclerosis Analyzed by a Computational Fluid Dynamics Model: A Pilot Study}

\author{
Zimo Chen 1,2, Haiqiang Qin 1,2,3,4, Jia Liu ${ }^{5}$, Bokai Wu ${ }^{5}$, Zaiheng Cheng ${ }^{5}$, Yong Jiang 1,2,3,4, \\ Liping Liu ${ }^{1,2,3,4}$, Lina Jing ${ }^{6}$, Xinyi Leng ${ }^{7}$, Jing Jing ${ }^{1,2,3,4}$, Yilong Wang ${ }^{1,2,3,4}$ and \\ Yongjun Wang ${ }^{1,2,3,4 *}$ \\ ${ }^{1}$ Department of Neurology, Beijing Tiantan Hospital, Capital Medical University, Beijing, China, ${ }^{2}$ China National Clinical \\ Research Center for Neurological Diseases, Beijing, China, ${ }^{3}$ Center of Stroke, Beijing Institute for Brain Disorders, Beijing, \\ China, ${ }^{4}$ Beijing Key Laboratory of Translational Medicine for Cerebrovascular Disease, Beijing, China, ${ }^{5}$ Shenzhen Institutes of \\ Advanced Technology, Chinese Academy of Sciences, Shenzhen, China, ${ }^{6}$ Department of Radiology, Beijing Tiantan Hospital, \\ Capital Medical University, Beijing, China, ${ }^{7}$ Department of Medicine and Therapeutics, The Chinese University of Hong Kong, \\ Prince of Wales Hospital, Shatin, China
}

Background: Although wall shear stress (WSS) and pressure play important roles in plaque vulnerability, characteristics of the two indices in intracranial atherosclerosis (ICAS) have not been fully investigated yet. This study aimed to elucidate this issue by means of establishing a non-invasive computational fluid dynamics method with time-of-flight magnetic resonance angiography (TOF-MRA) of the whole cerebral artery.

Materials and Methods: Subjects with symptomatic ICAS in the middle cerebral artery domain were enrolled, excluding those with concomitant internal carotid artery stenosis. Based on patient-specific TOF-MRA images for three-dimensional (3D) meshes and arterial blood pressure with patient-specific carotid artery ultrasonography for inlet boundary conditions, patients' three-dimensional hemodynamics were modeled by a finite element method governed by Navier-Stokes equations.

Results: Among the 55 atherosclerotic lesions analyzed by this TOF-MRA based computational fluid dynamics model, the maximum WSS (WSS $\max$ ) was most frequently detected at the apex points and the upper half of the upstream sections of the lesions, whereas the maximum pressure was most often located at the lower half of the upstream sections. As the percent stenosis increases, the relative value of WSS $\max$ and pressure drop increased with significantly increasing steep beyond 50\% stenosis. Moreover, WSS $_{\max }$ was found to linearly correlate with pressure drop in ICAS.

Conclusions: This study on ICAS revealed certain trends of longitudinal distribution of WSS and pressure and the influences of percent stenosis on cerebral hemodynamics, as well as the correlations between WSS and pressure drop. It represents a step forward in applying computational flow simulation techniques in studying ICAS and stroke, in a patient-specific manner.

Keywords: intracranial atherosclerosis, cerebral hemodynamics, wall shear stress, pressure, mathematical modeling, magnetic resonance angiography 


\section{INTRODUCTION}

Globally, stroke is a leading cause of mortality, disability, and the economic costs of treatment (1). Therein, intracranial atherosclerosis (ICAS) has been recognized as one of the most common causes of ischemic stroke and accounts for a majority of stroke recurrence, contributing to $30-50 \%$ of ischemic stroke and transient ischemic attack in Asian $(2,3)$. Despite optimal medical treatment, the remaining high risk of stroke in ICAS patients still lies in the limited understanding of underlying pathogenesis and that pathophysiological significance of ICAS cannot be completely reflected by anatomical severity, especially for mild and moderate stenosis (4).

One of the potential mechanisms is identified as the destabilizing effect by hemodynamic forces acting on plaques, generated by cerebral blood flow, the major indices of which are wall shear stress (WSS) and pressure (5). Thus, high mechanical load generated by hemodynamic forces often reveals a hemodynamic pattern prone to plaque rupture. In theory, high WSS may impose higher risk of plaque rupture, whereas low WSS is associated with the formation of plaques. In addition, pressure and the resultant translesional "pressure drop" also significantly affect the plaque vulnerability due to its considerable contribution to the total mechanical load as compared with WSS (5). It is therefore of great significance to clarify the characteristics of WSS and pressure of ICAS, in view of risk evaluation. Despite these speculations, data remain scarce by far on this issue.

Several studies have applied computational fluid dynamics (CFD) to simulate and analyze the hemodynamics of ICAS (6-8). However, these studies relied on images of computed tomography angiography, which causes concerns in radioactivity and contrast administration, and applied with the hypothetical inlet flow condition. In contrast, time-of-flight magnetic resonance angiography (TOF-MRA) is both radiation- and contrast-free $(9,10)$.

Thus, in this study, we aimed to investigate the characteristics of WSS and pressure through the non-invasive CFD model based on TOF-MRA and patient-specific inlet flow condition.

\section{MATERIALS AND METHODS}

\section{Patients}

Patients were screened and selected from a registry study conducted in our center. Subjects aging 18-80 years with symptomatic ICAS in middle cerebral artery (MCA) domain within 7 days after the symptom onset were included, excluding those with concomitant internal carotid artery stenosis. All subjects must have finished the examinations of brain TOFMRA and carotid artery ultrasonography, with velocity and diameter information available. Percent stenosis on TOF-MRA was calculated according to Warfarin-Aspirin Symptomatic Intracranial Disease trial (11). This cross-sectional study was approved by the ethics committee of Beijing Tiantan Hospital

Abbreviations: $\mathrm{M}_{\mathrm{up}}$, the middle point of the upstream section; $\mathrm{M}_{\mathrm{down}}$, the middle point of the downstream section. according to the principles expressed in the Declaration of Helsinki. All patients signed a written informed consent form.

\section{MRI}

Three-dimensional (3D) TOF-MRA was performed with a 3.0T MR scanner (Trio-Tim; Siemens, Erlangen, Germany), equipped with a maximal slew rate of $200 \mathrm{mT} \cdot \mathrm{m}^{-1} \cdot \mathrm{ms}^{-1}$, a maximal gradient strength of $45 \mathrm{mT} / \mathrm{m}$, a repetition time/echo time of $28 / 3.04 \mathrm{~ms}$, a field of view of $20 \times 18 \mathrm{~cm}^{2}$, matrix of $256 \times 179$, thickness of $0.7 \mathrm{~mm}$, slices per slab of 40 , and flip angle of $13^{\circ}$.

\section{CFD Modeling}

A personal workstation was used to process source images of TOF-MRA, and the data were stored in standard Digital Imaging and Communications in Medicine format. The 3D geometric model was then reconstructed by a suite of software, Mimics (Materialize NV, Belgium), and then examined and manually revised by two neurologists, with the primary collaterals included. Further processing of the vascular surface and generation of the computational domain (volume mesh) was performed using the ANSYS ICEM CFD meshing software (ANSYS, Inc., USA) (6-8).

Considering the complexity of the cerebral artery geometry, we used the unstructured tetrahedral cell for domain discretization, and the mesh was finer nearby the area of stenosis where the flow field was of greater interest (6). The total number of elements was above one million, and the minimum volume of the elements was about $1.0 \times 10^{-8} \mathrm{~cm}^{3}$ to capture the small-scale feature of the blood flow. The blood flow was assumed to be a viscous and incompressible Newtonian fluid. The blood parameters were defined by constant density $\rho=1.06 \times 10^{-3} \mathrm{~kg} \cdot \mathrm{m}^{-1}$ and constant dynamic viscosity $\mu=3.5 \times 10^{-3} \mathrm{~kg} \cdot \mathrm{m}^{-1} \cdot \mathrm{s}^{-1}$.

Based on the data of carotid artery ultrasonography, we calculated the blood flow rate as the inlet condition with the formula: blood flow rate $=$ mean velocity $\times$ cross-sectional area, where mean velocity was obtained using the value: $1 / 3$ peaksystolic velocity $+2 / 3$ end-diastolic velocity. We then applied an instantaneous pulsatile inflow and incorporated a lumped parameter model to mimic the downstream effects from the distal vessels on the outlet boundaries. The detailed calculation of the outlet condition was performed by the same method reported previously (8). The governing equations of blood flow were described by 3D steady incompressible Navier-Stokes equations. This model was solved by a parallel computer with $240 \mathrm{CPU}$ cores using the Newton-Krylov-Schwarz method.

An example of the hemodynamic simulation is shown in Figure 1. In this case, we included the bilateral anterior cerebral arteries and the posterior arteries into the CFD model where anterior communicating artery and right posterior communicating artery can be observed from the TOF-MRA scan.

\section{Measurements and Definition of the Specific Indices of WSS and Pressure}

We used ParaView software (5.5.0 64-bit) (Kitware Inc., USA) for extraction and measurement of WSS and pressure from the simulated CFD models. For each lesion, the measurement was 

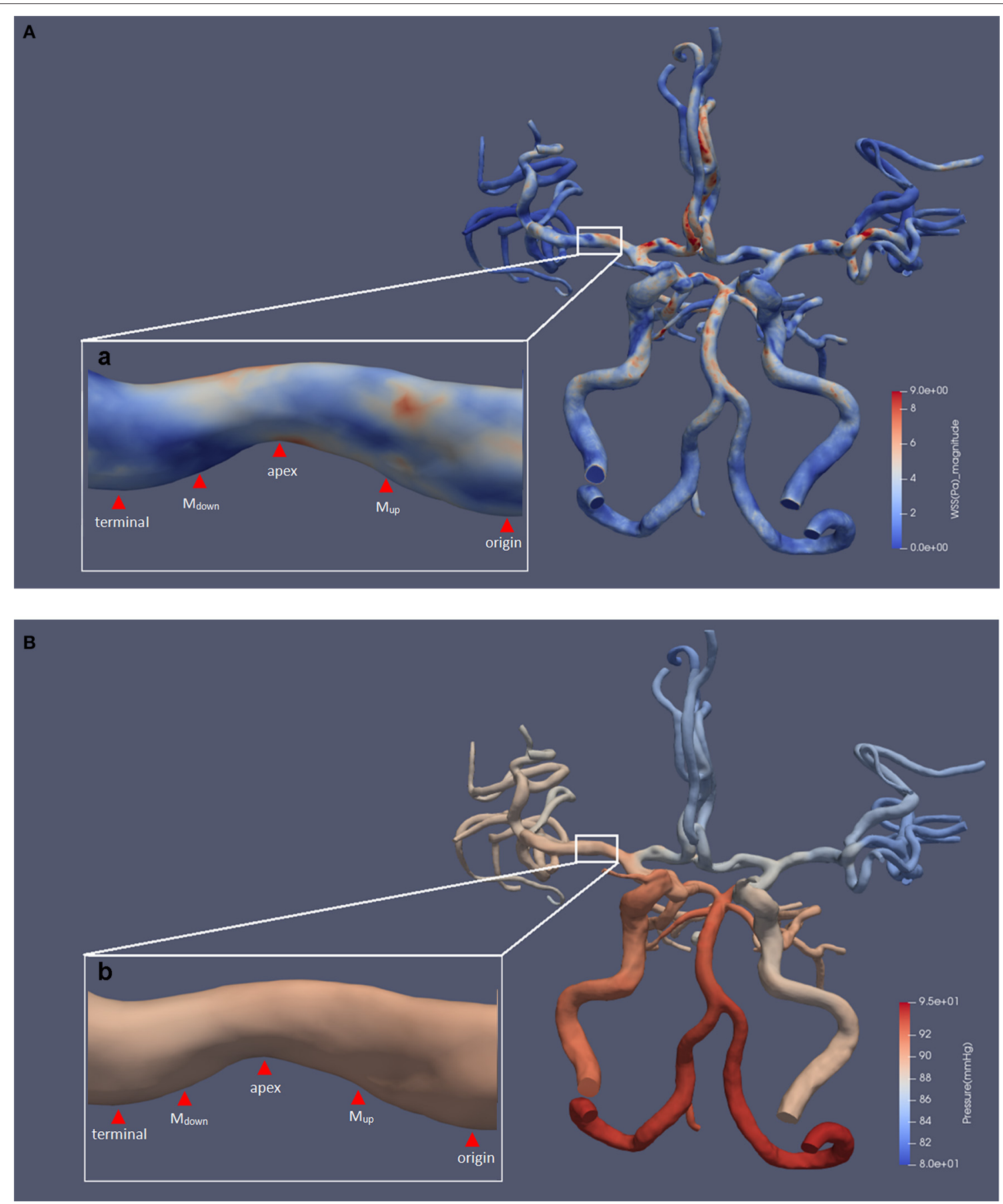

FIGURE 1 | The results of a typical hemodynamic simulation. The five defined points of the prominent side are shown. Panels (A) and (B), respectively, show the results of WSS and pressure contour maps. Subfigures (a) and (b) show an area of 34.3\% luminal stenosis in the right MCA M1 segment. We chose the posterior wall of the MCA as the prominent side of the lesion for measurement. The WSS $\max$ was located at the upper half of upstream section, and the magnitude was 7.47 pa. The WSS $_{\text {min }}$ was located at the downstream section, and the magnitude was 0.33 pa. The pressure ratio(termianl/origin) was 0.99 . MCA, middle cerebral artery; WSS, wall

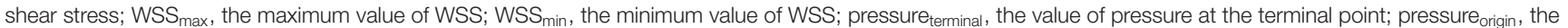
value of pressure at the origin point.

performed at the most severely narrowed longitudinal section of the diseased MCA, as shown in Figure 1, in accordance with previous studies on carotid and coronary arteries $(12,13)$.
Then, the prominent sides of lesions were defined by a greater contribution to the total luminal stenosis compared with the other side. We defined five measuring points along the 
longitudinal axis of the prominent side, as shown in Figure 1. The first and the last measuring points were located at the entrance and exit of the stenosis and were defined as the origin point (origin) and the terminal point (terminal). The tip of the lesion was then defined as the apex. Two more measuring points were located at the middle point of the origin-to-apex and the apexto-terminal sections, which were defined as "the middle point of upstream section" $\left(\mathrm{M}_{\mathrm{up}}\right)$ and "the middle point of downstream section" $\left(M_{\text {down }}\right)$, respectively. We further defined the maximum and minimum values of WSS and pressure on the prominent side as $\mathrm{WSS}_{\max }, \mathrm{WSS}_{\min }$, pressure max $_{\text {max }}$, and pressure min $_{\text {min }}$, respectively.

Due to the inlet flow conditions estimated on a patientspecific basis, the consequent hemodynamic parameters are determined by inlet flow conditions partly, which could cause difficulty in analyzing their relations with percent stenosis under the individualized inlet flow conditions. We therefore normalized them to achieve the relative indices such as WSS

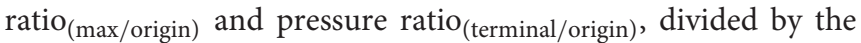
value of corresponding origin point, in order to attenuate the individual variation. In addition, we also used the indices of pressure drop (origin-to-terminal) to gauge the absolute changes of pressure across the entire lesion, calculated as pressure origin $_{\text {. }}$ subtracted by pressure terminal $_{\text {. }}$

All measurements, as shown in Figure 1, were performed by two experienced neurologists, and the values were then averaged. In order to minimize the possible hemodynamic interferes, tandem lesions with in-between distance $<2 \mathrm{~cm}$ and lesions located at the arterial bifurcation or at the opening of a branch were excluded from the measurements $(14,15)$.

\section{Statistical Analysis}

Categorical variables were expressed as absolute or relative frequencies. Age and percent stenosis were described using the means \pm SD. Indices of WSS and pressure were described using medians (interquartile range). The kappa statistics was performed to calculate the intra/inter-observation agreement between the two neurologists for the identification of atherosclerotic lesions. To compare WSS, pressure, and pressure drop between different locations, we used the Friedman Test for repeated measurements and the Bonferroni correction for multiple comparisons. Comparisons of proportions were calculated using the chi-square test. Spearman's rank correlation was applied to evaluate the correlations between percent stenosis and specific indices, as well as the correlations between indices of hemodynamic forces, with adjustment of age and sex.

Two-sided $p$-values of 0.05 were considered statistically significant. All analyses were performed with SAS 9.4 (SAS Institute, Cary, NC, USA).

\section{RESULTS}

Overall, 55 lesions within the MCA domains selected from 22 patients (mean age, 65.5 years; 54.5\% males) were included into the current analysis. For identification of atherosclerotic lesions, the intra/inter-observation agreements between the two neurologists were 0.877 and 0.815 , respectively. The mean value of the percent stenosis of these lesions was 43.2\% ( $\pm 17.3 \%)$. Specifically, $26(47.3 \%)$ lesions were located in M1 segments, and $24(43.6 \%)$ lesions were percent stenosis $\geq 50 \%$. Patients' demographic information is shown in Table S1.

\section{Description of the Magnitude of WSS and Pressure in Defined Points and Sections}

WSS and pressure were longitudinally asymmetric across lesions in general, and the variability of the indices at each location was remarkable (Table S2). The Friedman Test showed that the differences of magnitude between indices of different locations were significant in general ( $p$ $<0.001)$. $\mathrm{WSS}_{\text {apex }}$ was the highest among the five points, followed by WSS $_{\text {Mup }}$ (Table S3). In terms of pressure, it dropped significantly from $M_{\text {up }}$ to $M_{\text {down }}$, with nonsignificant change between origin and $\mathrm{M}_{\mathrm{up}}$, as well as $\mathrm{M}_{\text {down }}$ and Terminal (Table S4). Accordingly, pressure drop from $M_{u p}$ to $M_{\text {down }}$ [pressure $\operatorname{drop}_{\text {(Mup-to-apex) }}$ and pressure $\left.\operatorname{drop}_{(\text {apex-to-Mdown })}\right]$ were the largest among the entire prominent side, with non-significant differences between each other (Table S5).

\section{Distribution of Specific Indices of WSS and Pressure}

The WSS $_{\max }$ was most commonly found at the apex points $(52.7 \%)$ and was also frequently located at the upper half of upstream sections $\left(\mathrm{M}_{\text {up }}\right.$-to-apex) (40.0\%), with no significant difference of this feature between the two subgroups of stenosis severity $(<50, \geq 50 \%)$. On the contrary, $\mathrm{WSS}_{\min }$ was commonly observed at the downstream sections and the terminal points (38.2 and $36.4 \%$, respectively), and it was also observed at the origin points $(20.0 \%)$.

The pressure $\max$ was almost all located at the origin points and the lower half of upstream sections (67.3 and 27.3\%, respectively). Similar to WSS, the major location of pressure $_{\min }$ was within the downstream sections (70.9\%) and terminal points (25.5\%). These observations were consistent across the two subgroups of stenosis severity. The results of statistical analysis and detailed information on distribution are shown in Table 1.

In addition, to verify whether the distribution of specific indices may vary between different plaque locations, we further stratified the plaques according to their locations of the upper side, lower side, and both sides. However, we observed no significant difference between the three subgroups of plaque locations (Table S6).

\section{Correlation Between Specific Indices and Percent Stenosis}

As shown in Table 2 and Figures $\mathbf{2 A , B}$, both the WSS $\mathrm{ratio}_{\text {(max/origin) }}$ (positively) and the pressure $\mathrm{ratio}_{\text {(terminal/origin) }}$ (negatively) changed quadratically with respect to the percent stenosis with an inflection point around 50\%. Subsequently, we divided the data into two groups according to the 


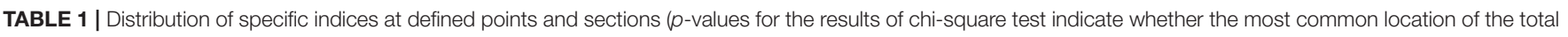
distribution becomes significantly different after grouping).

\begin{tabular}{|c|c|c|c|c|c|}
\hline \multirow{2}{*}{$\begin{array}{l}\text { Specific indices of } \\
\text { hemodynamic forces }\end{array}$} & \multirow{2}{*}{$\begin{array}{l}\text { Defined points and } \\
\text { sections }\end{array}$} & \multirow[t]{2}{*}{ Total } & \multicolumn{2}{|c|}{ Grouped by stenosis severity } & \multirow[t]{2}{*}{$p$-value } \\
\hline & & & Percent stenosis $\geq \mathbf{5 0} \%$ & Percent stenosis $<50 \%$ & \\
\hline \multirow[t]{4}{*}{ WSS $_{\max }$} & Apex & 29 (52.7\%) & $14(58.3 \%)$ & 15 (48.4\%) & 0.464 \\
\hline & $M_{\text {up }}$-to-apex & 22 (40.0\%) & $9(37.5 \%)$ & $13(41.9 \%)$ & \\
\hline & $M_{\text {down }}$ & $3(5.5 \%)$ & $1(4.2 \%)$ & $2(6.5 \%)$ & \\
\hline & Origin & $1(1.8 \%)$ & $0(0.0 \%)$ & $1(3.2 \%)$ & \\
\hline \multirow[t]{4}{*}{$W_{S S}$ min } & Downstream & $21(38.2 \%)$ & $9(37.5 \%)$ & 12 (38.7\%) & 0.927 \\
\hline & Terminal & 20 (36.4\%) & $10(41.7 \%)$ & $10(32.3 \%)$ & \\
\hline & Origin & $11(20.0 \%)$ & 5 (20.8\%) & $6(19.4 \%)$ & \\
\hline & Upstream & $3(5.4 \%)$ & $0(0.0 \%)$ & $3(9.6 \%)$ & \\
\hline \multirow[t]{5}{*}{ Pressure $_{\max }$} & Origin & 37 (67.3\%) & $17(70.8 \%)$ & $20(64.5 \%)$ & 0.620 \\
\hline & Origin-to- $\mathrm{M}_{\mathrm{up}}$ & $15(27.3 \%)$ & $7(29.2 \%)$ & $8(25.8 \%)$ & \\
\hline & Apex & $1(1.8 \%)$ & $0(0.0 \%)$ & $1(3.2 \%)$ & \\
\hline & Downstream & $1(1.8 \%)$ & $0(0.0 \%)$ & $1(3.2 \%)$ & \\
\hline & Terminal & $1(1.8 \%)$ & $0(0.0 \%)$ & 1 (3.2\%) & \\
\hline \multirow[t]{3}{*}{ Pressure $_{\min }$} & Downstream & 39 (70.9\%) & $16(66.7 \%)$ & $23(74.2 \%)$ & 0.542 \\
\hline & Terminal & 14 (25.5\%) & 8 (33.3\%) & $6(19.4 \%)$ & \\
\hline & Origin & $2(3.6 \%)$ & $0(0.0 \%)$ & $2(6.4 \%)$ & \\
\hline
\end{tabular}

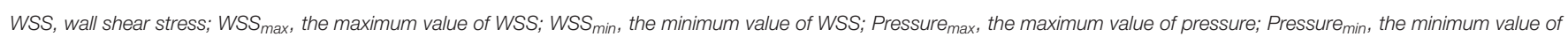
pressure; $M_{u p}$, the middle point of the upstream section; $M_{\text {down }}$, the middle point of the downstream section.

TABLE 2 | Results of Spearman's rank correlation between indices of hemodynamic forces and percent stenosis.

\begin{tabular}{lccrc}
\hline $\begin{array}{l}\text { Indices of } \\
\text { hemodynamic } \\
\text { forces }\end{array}$ & $\begin{array}{c}\text { Percent } \\
\text { stenosis }\end{array}$ & $\begin{array}{c}\text { Median (range } \\
\text { interquartile) }\end{array}$ & $\boldsymbol{p}$-Value & $\boldsymbol{r}_{\mathrm{s}}$ \\
\hline WSS ratio(max/origin) & $<50 \%$ & $2.61(1.91,3.20)$ & $<0.001$ & 0.598 \\
& $\geq 50 \%$ & $4.86(3.86,7.94)$ & $<0.001$ & 0.779 \\
Pressure & $<50 \%$ & $0.99(0.96,0.995)$ & 0.003 & -0.522 \\
ratio $_{\text {(terminal/origin) }}$ & $\geq 50 \%$ & $0.81(0.53,0.91)$ & $<0.001$ & -0.747 \\
& - & $0.47(0.66,0.87)$ & 0.651 & 0.063 \\
WSS ratio (min/origin) & & & & \\
\hline
\end{tabular}

WSS, wall shear stress; WSS $S_{\text {max }}$, the maximum value of WSS; WSS $S_{\text {min }}$, the minimum value of WSS; WSS origin, the value of WSS at the origin point; pressureterminal, the value of pressure at the terminal point; pressure origin, the value of pressure at the origin point.

$50 \%$ stenosis and performed Spearman's rank correlation for the two groups, respectively. Adjusted by age and sex, the correlations were significant for the two indices. Furthermore, both of the indices showed different coefficients for the two stenosis severity, with larger $r_{\mathrm{s}}$ of stenosis $\geq 50 \%$ subgroups, compared with the stenosis $<50 \%$ subgroups. For WSS ratio $_{(\min / \text { origin) }}$, we did not find the correlation to be significant (Figure 2C).

As shown in Figures $\mathbf{3 A}, \mathbf{B}$, the magnitude and distribution of WSS and pressure varied with aggravation of ICAS, percent stenosis of which was $47.1,56.3$, and $70.0 \%$ for subfigures a-c, respectively.

\section{Correlation Between WSS max $_{\text {, Pressure }}$ Ratio(terminal/origin), and Pressure Drop (origin-to-terminal)} Furthermore, by this TOF-MRA-based CFD model, we also evaluated the correlation between indices of hemodynamic forces. Figures $\mathbf{4 A , B}$, show the correlation between $\mathrm{WSS}_{\max }$,

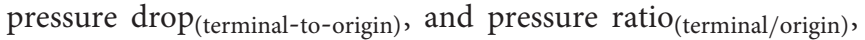
with adjustment of age and sex. Therein, we observed a linear relationship between $\mathrm{WSS}_{\max }$ and the absolute and relative value of pressure drop, as indicated by the coefficients $\left[r_{\mathrm{s}}=0.893(p<\right.$ $0.001)$ and $r_{\mathrm{s}}=-0.879(p<0.001)$, respectively].

\section{DISCUSSION}

In this study, we proposed the potential value of routine TOFMRA-based cerebral blood flow simulation in hemodynamic assessment of ICAS. For WSS and pressure, we observed the certain trends of longitudinal distribution and changes around atherosclerotic lesions, as well as the correlation between WSS and pressure drop. Notably, the progression of stenosis severity had a significant impact on the magnitude of WSS and pressure, especially when percent stenosis $\geq 50 \%$.

Previous studies on carotid artery revealed that plaques often ruptured at certain regions under high WSS (16-19), suggesting that the rupture of plaques may be attributed to the high shear rate of blood flow at the region of most severe stenosis. Our study also showed that WSS $_{\max }$ mostly occurred within 

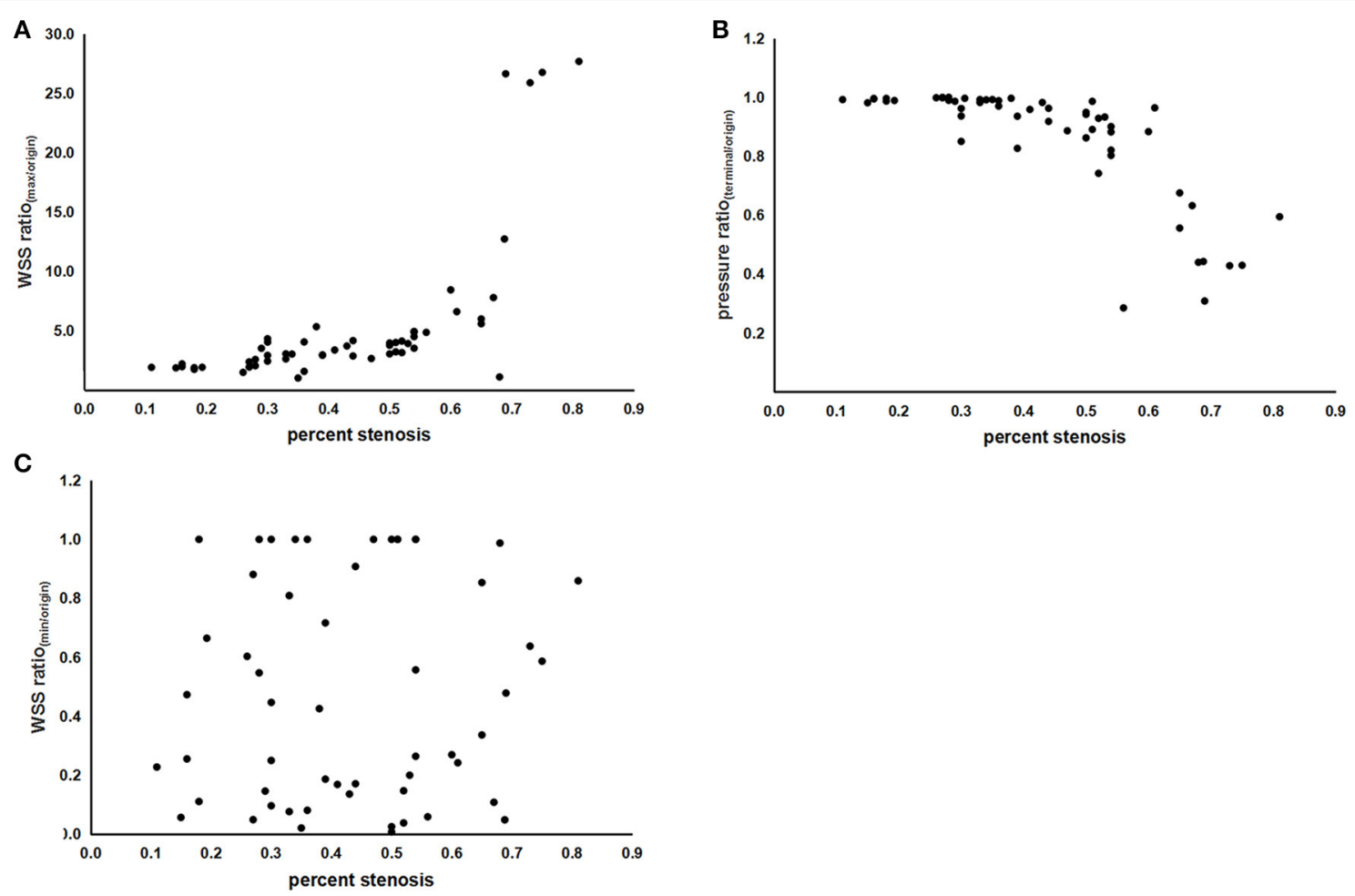

FIGURE 2 | The scatterplots of correlations between indices of hemodynamic forces and percent stenosis. (A) Correlation between the WSS ratio(max/origin) and percent stenosis. (B) Correlation between the pressure ratio(terminal/origin) and percent stenosis. (C) Correlation between the WSS ratio(min/origin) and percent stenosis.

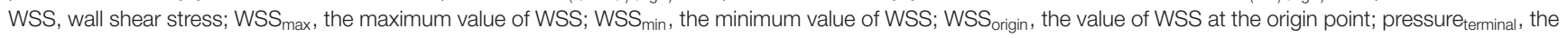

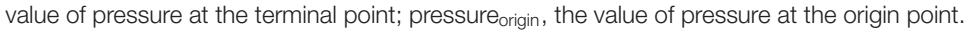

the upper half of upstream sections including the apex points, probably indicating the certain region with high risk of plaque rupture in ICAS. One previous study reported that low WSS could induce the differentiation of an atherogenic endothelial phenotype and the formation of early atherosclerotic lesion through the regulation of endothelial gene expression (20). From our investigation, we observed that $\mathrm{WSS}_{\min }$ correlated with the downstream section of atherosclerotic lesion in space. Due to the previous study revealing that coronary plaque deposition frequently occurs near low WSS regions (21), this observation indicates the possibility that $\mathrm{WSS}_{\min }$ may also involve in the ICAS progression, in the direction from the proximal to distal side.

Besides WSS in tangential direction, as the hemodynamic force acting perpendicularly to the lateral wall, pressure also plays an important role in plaque destabilization (5). It has been reported that upstream-ruptured plaques in the carotid artery were associated with a higher pressure drop between the upstream and the downstream shoulder sections (22). The current study showed that the upper half section (between $\mathrm{M}_{\text {up }}$ and $\mathrm{M}_{\text {down }}$ ) sustained the majority of total pressure drop, implying that this region carries a significantly larger mechanical load and may be at a higher risk of plaque rupture in ICAS.

Predicting the critical indices of hemodynamic forces such as $\mathrm{WSS}_{\max }$ and pressure drop, according to the progression of stenotic severity, can be beneficial for assessing the functional severity of ICAS. We found that the indices of WSS $\mathrm{ratio}_{(\mathrm{max} / \text { origin) }}$ and pressure $\mathrm{ratio}_{(\text {terminal/origin) }}$ showed a significant correlation with percent stenosis, in a non-linearly proportional manner. Moreover, the scatterplots and the $r_{\mathrm{s}}$ for each subgroup indicated a threshold effect for the two indexes, suggesting that the relative value of $\mathrm{WSS}_{\max }$ and pressure drop may increase with percent stenosis in a more dramatic way at $\geq 50 \%$ diameter stenosis. This threshold effect was in accordance with the finding that symptomatic ICAS patients with percent stenosis $\geq 50 \%$ had higher risk of stroke recurrence compared to those with percent stenosis $<50 \%$ (4). Our results explain the finding from a mechanical viewpoint that percent stenosis significantly influences the hemodynamic forces in ICAS patients, leading to consequent increasing risk of plaque rupture, especially when percent stenosis is $\geq 50 \%$, indicating the necessity to conduct hemodynamic assessment among this population.

It is worth noting that the index of pressure ratio(terminal/origin) in our study was similar to the recently proposed index of fractional pressure ratio by Liu et al., who have demonstrated that for symptomatic ICAS, it was a good approximation of fractional flow reserve, the gold standard for assessing the physiological significance of coronary stenotic lesions $(8,23)$. Moreover, one recent study has demonstrated the close correlation between translesional pressure ratio, translesional WSS ratio, and stroke 

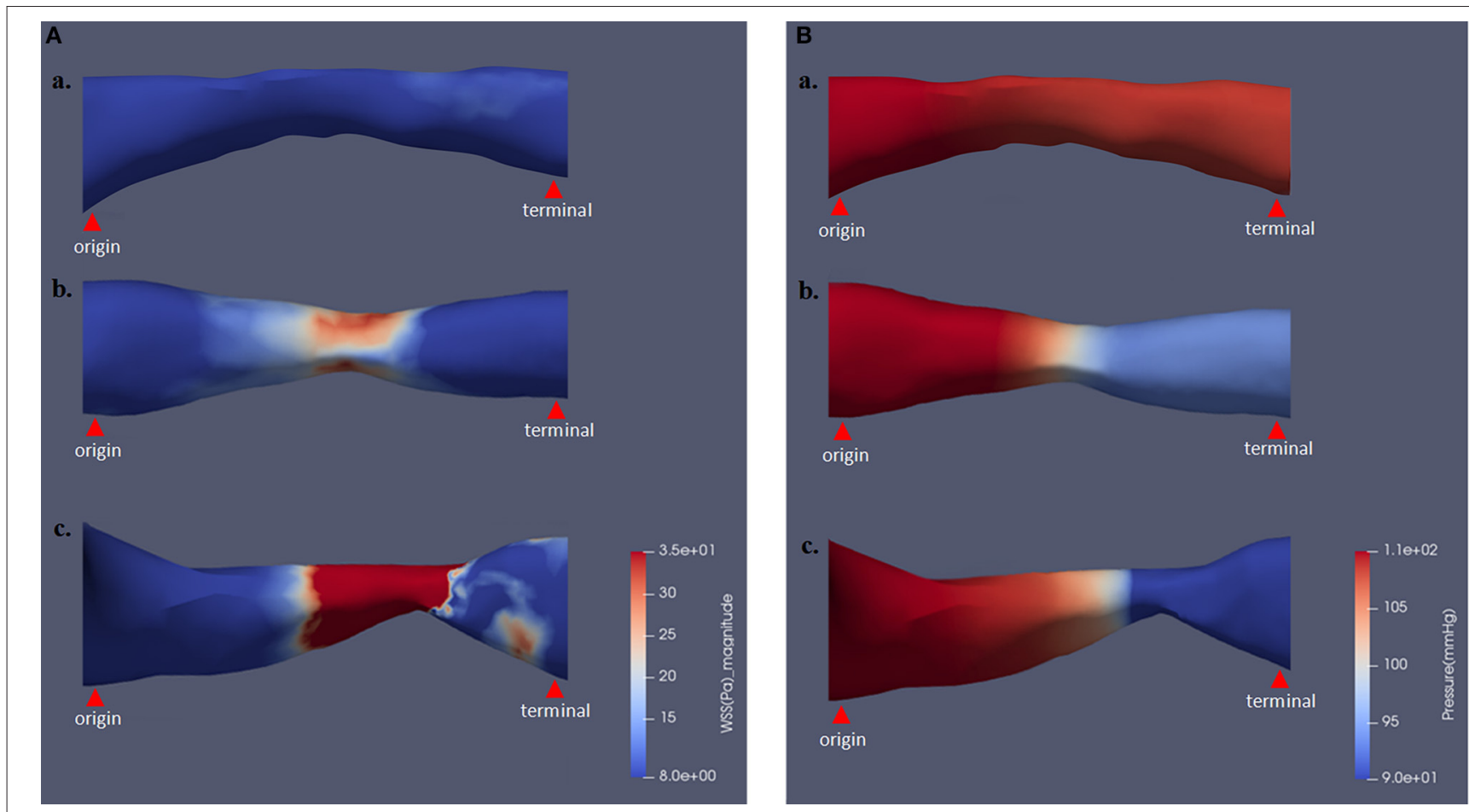

FIGURE 3 | The magnitude and distribution of WSS and pressure varying with different stenosis severity. Panels (A) and (B), respectively, show the results of WSS and pressure contour maps. Percent stenosis values of subfigures subfigures (a) and (b) were $47.1,56.3$, and $70.0 \%$, respectively. WSS, wall shear stress.
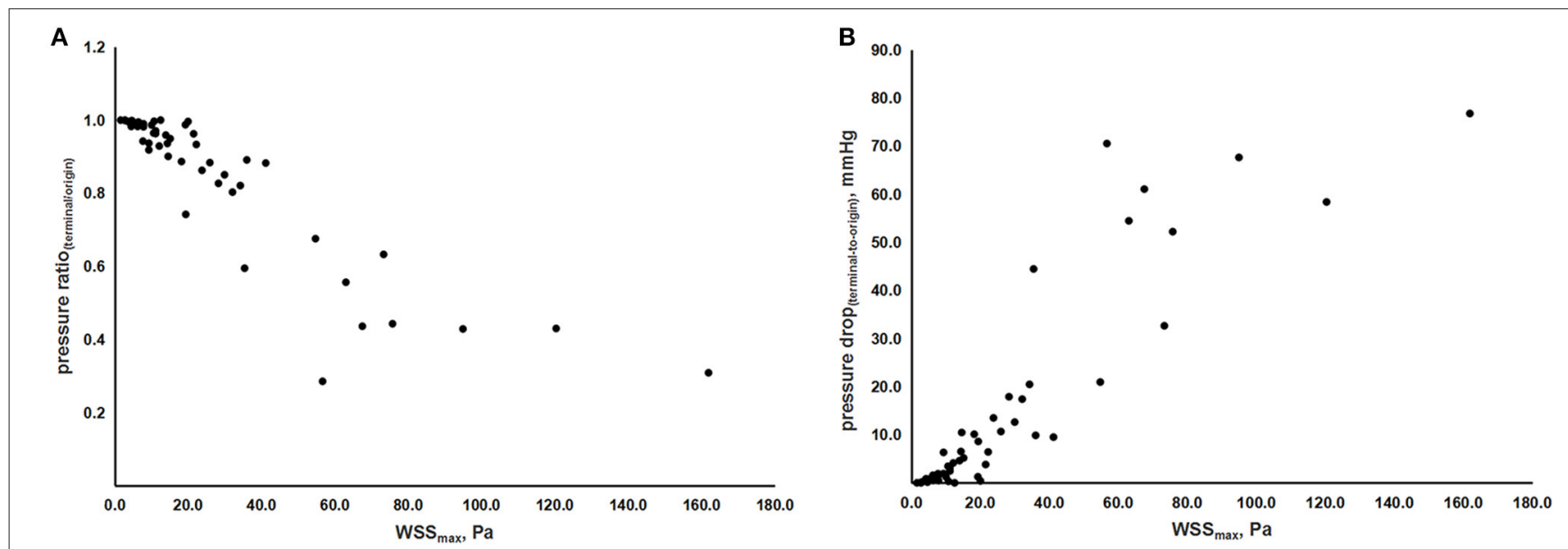

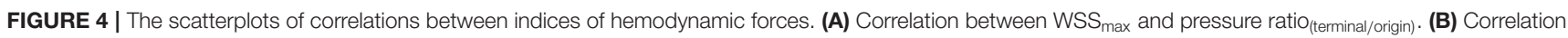
between WSS $_{\max }$ and pressure drop (origin-to-terminal). WSS, wall shear stress; WSS max $_{\text {, the maximum value of WSS; pressure }}$ terminal, the value of pressure at the terminal

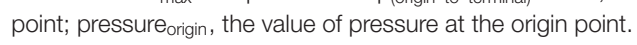

relapse in symptomatic ICAS patients (6). Our study initially demonstrates that the two indexes were not altered significantly until stenosis severity reached $50 \%$. Furthermore, the scatterplots also show the inter-subject variability, indicating the fact that the hemodynamic damage of ICAS was partly determined by percent stenosis, as proved by previous studies on coronary heart disease $(24,25)$. Other factors may also involve, such as the presence of collaterals and the plaque characteristics (26).
From the proposed TOF-MRA-based CFD model, we also found that the $\mathrm{WSS}_{\max }$ was linearly correlated with pressure ratio(terminal/origin) $_{\text {(n) }}$ and pressure drop $_{\text {(terminal-to-origin) }}$ in ICAS, accordingly with the result in coronary artery (27). Since WSSmax values beyond a critical value can induce atherosclerotic inflammation (6), our results also indicated that the plaque vulnerability induced by WSS $_{\max }$ may be accompanied with the aggravation of mechanical load, and there was a possibility 
that plaque vulnerability and hypoperfusion collaboratively participate in the risk of stroke in ICAS.

In the current study, we demonstrated the utility of routine performed TOF-MRA in hemodynamic assessment of ICAS. The decreased sensitivity of TOF-MRA to disturbed and slow flow has been shown, which may limit its application in severe stenosis (28). Instead of the hypoperfusion in severe stenosis, plaque rupture caused by mechanical destruction acts as the major pathological process of stroke in mild to moderate stenosis of ICAS, and it is therefore of clinical value to detect plaque vulnerability, in other words, the imbalance between plaque strength and hemodynamic forces imposing on plaques (29). However, in spite of the high-resolution MRI for evaluation of plaque composition, there is still a lack of methods to elucidate the other aspect. From this perspective, this proposed TOFMRA-based CFD model can be appropriate for risk evaluation, especially for mild to moderate stenosis.

This study has certain limitations. First, the measurements performed in a plane inherently ignore the plaque morphology out of the chosen plane, which may affect the obtained indices. Second, there is still a lack of established method to calculate the mean blood flow volume of precerebral arteries as the inlet condition. The computation method we used to calculate mean velocity referred to the calculation of mean arterial pressure to achieve an approximate value of timeaveraged mean flow velocity was in accordance with previous studies $(30,31)$. Third, to only evaluate one of the aspects involving in the process of plaque rupture such as the external mechanical forces of WSS and pressure cannot fully address the risk of stroke, especially in the absence of plaque properties reflected by high-resolution MRI, which also prevented us from identifying the atheromatous lesions in a more rigorous way (32). Therefore, to further perform the risk prediction, the evaluation of hemodynamics based on high-resolution MRI is desired from further studies. Fourth, the sample size of this study is relatively small. However, it has clarified the characteristics of hemodynamic forces of ICAS and the nonlinear relationship between percent stenosis and the specific indices, which can be verified with larger sample sizes in the future.

\section{CONCLUSIONS}

In this study, we performed patient-specific description of the magnitude and distribution of WSS and pressure in realistic geometries of ICAS, which has the potential value to facilitate more accurate risk stratification and more effective prevention for ICAS patients. Meanwhile, we demonstrated the feasibility of a novel radiation- and contrast-free method using routine TOF-MRA-based CFD models, to non-invasively

\section{REFERENCES}

1. GBD 2016 Stroke Collaborators. Global, regional, and national burden of stroke, 1990-2016: a systematic analysis for the Global quantify hemodynamic indices of ICAS. Further studies need to verify the roles of hemodynamic forces in governing the stroke risk in ICAS patients and to test indices of the TOF-MRA-based CFD model on accuracy.

\section{DATA AVAILABILITY STATEMENT}

The datasets generated for this study are available on request to the corresponding author.

\section{ETHICS STATEMENT}

This study was approved by the ethics committee of Beijing Tiantan Hospital according to the principles expressed in the Declaration of Helsinki. The relevant Judgement's reference number is: KYSB2016-147.

\section{AUTHOR CONTRIBUTIONS}

YoW, YiW, and LL designed the research protocol and proposed the method of measurement for hemodynamic forces. JL and HQ proposed and designed the CFD model for this study. ZChen wrote the manuscript. HQ and JJ collected the MRA images. BW, ZCheng, XL, and ZChen reconstructed the 3D artery from the MRA images and transferred the 3D artery into meshes. LJ and HQ performed the measurement of hemodynamic forces. YJ and ZChen performed the statistical analysis.

\section{FUNDING}

This study was supported by the Ministry of Science and Technology of China (Grant Nos. 2016YFC1301604 and 2016YFC1301602) and the Natural Science Foundation of China (Grant No. 81870907).

\section{ACKNOWLEDGMENTS}

We would like to convey our appreciation to all patients participating in this study and the staff of the Shenzhen Institutes of Advanced Technology, Chinese Academy of Sciences, Shenzhen, China, and the staff of the radiology department, Beijing Tiantan Hospital, Capital Medical University, Beijing, China.

\section{SUPPLEMENTARY MATERIAL}

The Supplementary Material for this article can be found online at: https://www.frontiersin.org/articles/10.3389/fneur. 2019.01372/full\#supplementary-material
Burden of Disease Study 2016. Lancet Neurol. (2019) 18:439-58. doi: 10.1016/S1474-4422(19)30034-1

2. Wang YJ, Zhao XQ, Liu LP, Soo YO, Pu Y, Pan Y, et al. Prevalence and outcomes of symptomatic intracranial large artery stenoses and occlusions in 
China: the Chinese Intracranial Atherosclerosis (CICAS) Study. Stroke. (2014) 45:663-9. doi: 10.1161/STROKEAHA.113.003508

3. Chimowitz M, Lynn M, Howlett-Smith H, Stern BJ, Hertzberg VS, Frankel MR, et al. Warfarin-Aspirin Symptomatic intracranial disease trial investigators. Comparison of warfarin and aspirin for symptomatic intracranial arterial stenosis. $N$ Engl J Med. (2005) 352:1305-16. doi: 10.1056/NEJMoa043033

4. Kasner SE, Chimowitz MI, Lynn MJ, Koo JS, Lee JH, Park JM, et al. Predictors of ischemic stroke in the territory of a symptomatic intracranial arterial stenosis. Circulation. (2006) 113:555-63. doi: 10.1161/CIRCULATIONAHA.105.578229

5. Li ZY, Taviani V, Tang T, Sadat U, Young V, Patterson A, et al. The mechanical triggers of plaque rupture: shear stress vs pressure gradient. Br J Radiol. (2009) 82:S39. doi: $10.1259 / \mathrm{bjr} / 15036781$

6. Leng X, Lan L, Ip HL, Abrigo J, Scalzo F, Liu H, et al. Hemodynamics and stroke risk in intracranial atherosclerotic disease. Ann Neurol. (2019) 85:752-64. doi: 10.1002/ana.25456

7. Leng X, Scalzo F, Ip HL, Johnson M, Fong AK, Fan FS, et al. Computational fluid dynamics modeling of symptomatic intracranial atherosclerosis may predict risk of stroke recurrence. PLoS ONE. (2014) 9:e97531. doi: 10.1371/journal.pone.0097531

8. Liu J, Yan Z, Pu Y, Shiu WS, Wu J, Chen R, et al. Functional assessment of cerebral artery stenosis: a pilot study based on computational fluid dynamics. $J$ Cereb Blood Flow Metab. (2017) 37:2567-76. doi: 10.1177/0271678X16671321

9. Heiserman JE, Drayer BP, Keller PJ, Fram EK. Intracranial vascular stenosis and occlusion: evaluation with three-dimensional time-offlight MR angiography. Radiology. (1992) 185:667-73. doi: 10.1148/ radiology.185.3.1438743

10. Korogi Y, Takahashi M, Mabuchi N, Miki H, Shiga H, Watabe T, et al. Intracranial vascular stenosis and occlusion: diagnostic accuracy of threedimensional, Fourier transform, time-of-flight MR angiography. Radiology. (1994) 193:187. doi: 10.1148/radiology.193.1.8090890

11. Samuels OB, Joseph GJ, Lynn MJ, Smith HA, Chimowitz MI. A Standardized method for measuring intracranial arterial stenosis. Ajnr Am J Neuroradiol. (2000) 21:643-6.

12. Thrysøe SA, Oikawa M, Yuan C, Eldrup N, Klaerke A, Paaske $\mathrm{WP}$, et al. Longitudinal distribution of mechanical stresses in carotid plaques of symptomatic patients. Stroke. (2010) 41:1041-3. doi: 10.1161/STROKEAHA.109.571588

13. Choi G, Lee JM, Kim HJ, Park JB, Sankaran S, Otake H, et al. Coronary artery axial plaque stress and its relationship with lesion geometry: application of computational fluid dynamics to coronary CT angiography. JACC Cardiovasc Imaging. (2015) 8:1156-66. doi: 10.1016/j.jcmg.2015.04.024

14. Steinman DA, Poepping TL, Tambasco M, Rankin RN, Holdsworth DW. Flow patterns at the stenosed carotid bifurcation: effect of concentric versus eccentric stenosis. Ann Biomed Eng. (2000) 28:415-23. doi: 10.1114/1.279

15. Javadzadegan A, Yong AS, Chang M, Ng AC, Yiannikas J, Ng MK, et al. Flow recirculation zone length and shear rate are differentially affected by stenosis severity in human coronary arteries. Am J Physiol Heart Circ Physiol. (2013) 304:H559-66. doi: 10.1152/ajpheart.00428.2012

16. Groen HC, Gijsen FJ, van der Lugt A, Ferguson MS, Hatsukami TS, van der Steen AF, et al. Plaque rupture in the carotid artery is localized at the high shear stress region a case report. Stroke. (2007) 38:2379-81. doi: 10.1161/STROKEAHA.107.484766

17. Kock SA, Nygaard JV, Eldrup N, Fründ ET, Klaerke A, Paaske WP, et al. Mechanical stresses in carotid plaques using MRI-based fluid-structure interaction models. J Biomech. (2008) 41:1651-8. doi: 10.1016/j.jbiomech.2008.03.019

18. Li ZY, Howarth SP, Tang T, Graves MJ, U-King-Im J, Trivedi RA, et al. Structural analysis and magnetic resonance imaging predict plaque vulnerability: a study comparing symptomatic and asymptomatic individuals. J Vasc Surg. (2007) 45:768-75. doi: 10.1016/j.jvs.2006.12.065

19. Li ZY, Howarth S, Trivedi RA, U-King-Im JM, Graves MJ, Brown A, et al. Stress analysis of carotid plaque rupture based on in vivo high resolution MRI. J Biomech. (2006) 39:2611-22. doi: 10.1016/j.jbiomech.2005.08.022
20. Chatzizisis YS, Coskun AU, Jonas M, Edelman ER, Feldman CL, Stone PH. Role of endothelial shear stress in the natural history of coronary atherosclerosis and vascular remodeling: molecular, cellular, and vascular behavior. J Am Coll Cardiol. (2007) 49:2379-93. doi: 10.1016/j.jacc.2007.02.059

21. Cicha I, Wörner A, Urschel K, Beronov K, Goppelt-Struebe M, Verhoeven E, et al. Carotid plaque vulnerability: a positive feedback between hemodynamic and biochemical mechanisms. Stroke. (2011) 42:3502-10. doi: 10.1161/STROKEAHA.111.627265

22. Sui B, Gao P, Lin Y, Jing L, Sun S, Qin H. Hemodynamic parameters distribution of upstream, stenosis center, and downstream sides of plaques in carotid artery with different stenosis: a MRI and CFD study. Acta Radiol. (2015) 56:347-54. doi: 10.1177/0284185114526713

23. De Bruyne B, Pijls NH, Bartunek J, Kulecki K, Bech JW, De Winter H, et al. Fractional flow reserve in patients with prior myocardial infarction. Circulation. (2001) 104:157-62. doi: 10.1161/01.CIR.104.2.157

24. Bruyne BD, Sarma J. Fractional flow reserve: a review. Heart. (2008) 94:94 9-59. doi: 10.1136/hrt.2007.122838

25. Gaur S, Øvrehus KA, Dey D, Leipsic J, Bøtker HE, Jensen JM, et al. Coronary plaque quantification and fractional flow reserve by coronary computed tomography angiography identify ischaemia-causing lesions. Eur Heart $J$. (2016) 37:1220-7. doi: 10.1093/eurheartj/ehv690

26. Ng J, Bourantas CV, Torii R, Ang HY, Tenekecioglu E, Serruys PW, et al. Local hemodynamic forces after stenting: implications on restenosis and thrombosis. Arterioscler Thromb Vasc Biol. (2017) 37:2231-42. doi: 10.1161/ATVBAHA.117.309728

27. Lee KE, Kim GT, Lee JS, Chung JH, Shin ES, Shim EB. A patient-specific virtual stenotic model of the coronary artery to analyze the relationship between fractional flow reserve and wall shear stress. Int J Cardiol. (2016) 222:799-805. doi: 10.1016/j.ijcard.2016.07.153

28. Urchuk SN, Plewes DB. Mechanisms of flow-induced signal loss in MR angiography. J Magn Reson Imaging. (1992) 2:453-62. doi: 10.1002/jmri.1880020415

29. Truijman MT, de Rotte AA, Aaslid R, van Dijk AC, Steinbuch J, Liem MI, et al. Intraplaque hemorrhage, fibrous cap status, and microembolic signals in symptomatic patients with mild to moderate carotid artery stenosis: the Plaque at RISK study. Stroke. (2014) 45:3423-6. doi: 10.1161/STROKEAHA.114.0 06800

30. Dörfler P, Puls I, Schliesser M, Maurer M, Becker G. Measurement of cerebral blood flow volume by extracranial sonography. I Cereb Blood Flow Metab. (2000) 20:269-71. doi: 10.1097/00004647-20000200 0-00007

31. Schoning M, Scheel P. Color duplex measurement of cerebral blood flow volume: intra- and interobserver reproducibility and habituation to serial measurements in normal subjects. J Cereb Blood Flow Metab. (1996) 16:523-31. doi: 10.1097/00004647-19960500 0-00020

32. Sato $\mathrm{Y}$, Ogasawara $\mathrm{K}$, Narumi $\mathrm{S}$, et al. Optimal MR plaque imaging for cervical carotid artery stenosis in predicting the development of microembolic signals during exposure of carotid arteries in endarterectomy: comparison of 4 T1weighted imaging techniques. AJNR Am J Neuroradiol. (2016) 37:1146-54. doi: 10.3174/ajnr.A4674

Conflict of Interest: The authors declare that the research was conducted in the absence of any commercial or financial relationships that could be construed as a potential conflict of interest.

Copyright (๑) 2020 Chen, Qin, Liu, Wu, Cheng, Jiang, Liu, Jing, Leng, Jing, Wang and Wang. This is an open-access article distributed under the terms of the Creative Commons Attribution License (CC BY). The use, distribution or reproduction in other forums is permitted, provided the original author(s) and the copyright owner(s) are credited and that the original publication in this journal is cited, in accordance with accepted academic practice. No use, distribution or reproduction is permitted which does not comply with these terms. 